\title{
Distinct properties in ventral pallidum projection neuron subtypes
}

\author{
Nimrod Bernat ${ }^{1 *}$, Rianne Campbell ${ }^{2 *}$, Hyungwoo Nam², Mahashweta Basu ${ }^{3,4}$, Tal \\ Odesser $^{1}$, Gal Elyasaf ${ }^{1}$, Michel Engeln ${ }^{2,5}$, Ramesh Chandra², Shana Golden ${ }^{1}$, \\ Seth Ament ${ }^{3,4}$, Mary Kay Lobo ${ }^{2 \#}$, Yonatan M. Kupchik ${ }^{1 \#}$ \\ ${ }^{1}$ Department of Medical Neurobiology, Faculty of Medicine, The Institute for \\ Medical Research Israel-Canada, The Hebrew University of Jerusalem, Israel \\ 2Department of Anatomy and Neurobiology, University of Maryland School of \\ Medicine, Baltimore, MD, USA \\ ${ }^{3}$ Department of Psychiatry, University of Maryland School of Medicine, Baltimore, \\ MD, USA. \\ ${ }^{4}$ Institute for Genome Sciences, University of Maryland School of Medicine, \\ Baltimore, MD, USA. \\ 5University of Bordeaux, CNRS, IMN, UMR 5293, F-33000 Bordeaux, France. \\ ${ }^{*}$ Equal contribution \\ \#To whom correspondence should be addressed
}

\section{Abstract}

The ventral pallidum (VP), a major component of the basal ganglia, plays a critical role in motivational disorders. It sends projections to many different brain regions but it is not yet known whether and how these projections differ in their cellular properties, gene expression patterns, connectivity and role in reward seeking. In this study, we focus on four major outputs of the VP - to the lateral hypothalamus $(\mathrm{LH})$, ventral tegmental area (VTA), mediodorsal thalamus (MDT), and lateral habenula ( $\mathrm{LHb}$ ) - and examine the differences between them in 1) baseline gene expression profiles using projection-specific RNA-sequencing; 2) physiological parameters using whole-cell patch clamp; and 3) their influence on cocaine reward using chemogenetic tools. We show that these four VP efferents differ in all three aspects and highlight specifically differences between the projections to the LH and the VTA. These two projections originate largely from separate populations of neurons, express distinct sets of genes related to neurobiological functions, and show opposite physiological and behavioral properties. Collectively, our data 
demonstrates for the first time that VP neurons exhibit distinct molecular and cellular profiles in a projection-specific manner, suggesting that they represent different cell types.

\section{Introduction}

The ventral pallidum (VP) is a central hub in the basal ganglia and plays an important role in motivation, reward, and addiction ${ }^{1,2}$. The VP receives dense inhibitory input from the nucleus accumbens (NAc), however inputs from other sources such as the amygdala, thalamus and hypothalamus have also been reported ${ }^{1}$. The VP has wide-ranging downstream projections, including dense projections to the ventral tegmental area (VTA), the lateral hypothalamus (LH), the mediodorsal thalamus (MDT) and the lateral habenula $(\mathrm{LHb})$ among other targets ${ }^{1}$.Previous work has identified that VP projection neurons exert different roles in rewarding and aversive behaviors ${ }^{1,3-6}$. Despite these distinct roles in behavior, it is theorized that different VP projection neurons are composed of the same neuronal subtype, possibly even with significant overlap between the projection subpopulations. However, no direct comparisons of the cellular and molecular compositions within VP projection neurons have been performed.

The neuronal composition of the VP is not fully understood. Past work has categorized VP neurons based on their neurochemical content. Thus, most of the VP neurons are GABAergic, and a minority are glutamatergic and cholinergic ${ }^{1}$. However, other studies define VP neurons according to a neuropeptide they release ${ }^{7}$, or the expression of a particular receptor ${ }^{8}$ or protein $^{1,2,9,10}$. A full characterization of the celluar and molecular phenotypes within VP neurons is required to identify the cell-type and circuit-specific mechanisms relavent to psychiatric diseases.

In this work we examine whether the different projections of the VP to the VTA, LH, MDT and LHb differ in their physiological properties, baseline gene expression and behavioral responses to cocaine. Our data highlight the $V P_{\rightarrow V T A}$ and $V P \rightarrow L H$ projections as highly distinct in these parameters, suggesting these two projections of the VP may be different types of neurons.

\section{Methods}


Experimental subjects - For electrophysiology, labeling and behavioral experiments, subjects were naïve C57bl6/J wildtype male and female mice who were approximately 8 weeks old at the beginning of the experiments. A 12-hour reversed light/dark cycle was maintained, with the lights turned off at 8:00 am. Experimental procedures were conducted during dark hours. Mice were group-housed and nesting/enrichment material was available. Experimentation began after a minimum of 7 days of acclimation to animal facility. All experimental procedures were approved by the Authority for Biological and Biomedical Models in the Hebrew university. For projection-specific gene expression analysis, adult (7-8 weeks old) male RiboTag (RT)+/+ mice (Rpl22tm1.1Psam/J) on a C57BI/6 background were used. RiboTag mice were given food and water ad libitum throughout the study. These studies were conducted in accordance with guidelines of the Institutional Animal Care and Use Committees at University of Maryland School of Medicine.

Stereotaxic microinjections - Mice were anesthetized with isoflurane and fixed in a stereotaxic frame (Kopf, Model 940). Viruses or red Retrobeads ${ }^{\mathrm{TM}}$ (Lumafluor, Durham, North Carolina) were microinjected into one or more of the following structures - the MDT (coordinates in millimeters relative to Bregma: anterior/posterior -1.5 , medial/lateral \pm 0.47 , dorsal/ventral -3.63), LHb (A/P -1.8, M/L \pm 0.7 , D/N -2.91), LH (A/P -0.3, M/L $\pm 0.9, \mathrm{D} / \mathrm{V}-$ 5.5), VTA (A/P -2.9, M/L $\pm 0.5, D / V-4.53)$, VP (A/P 0.7, M/L $\pm 1.2, D / V-5.21)$ and NAc (A/P 1.8, $\mathrm{M} / \mathrm{L} \pm 1.0, \mathrm{D} / \mathrm{V}-4.6$ ) (see below for details on the injections in each experiment). Injections were performed bilaterally by drilling bilateral holes into the skull and then microinjecting the viral constructs [through a 33 ga NanoFil syringe (World Precision Instruments; $300 \mathrm{nl}$ per hemisphere, $100 \mathrm{nl} / \mathrm{min}$, needle retracted $5 \mathrm{~min}$ after injection terminated)] or Retrobeads [through a 30 ga syringe (Hamilton: $300 \mathrm{nl}$ per hemisphere, $300 \mathrm{nl} / \mathrm{min}$, needle retracted $5 \mathrm{~min}$ after injection terminated)] into the target structure. Circuit-specific gene expression analysis was performed using retrograde AAV-Cre virus in combination with Ribotag mice. Briefly, anesthetized RiboTag RT +/+ mice were bilaterally injected with AAV5-Cre viruses (AAV sterotype 5 AAV5.hSyn.HI.eGFPCre.WPRE.SV40 (Addgene; \#105540) at the following coordinates: ventral pallidum (from Bregma; $10^{\circ}$ angle, anterior/posterior: $\mathrm{AP}+0.9$, medial/lateral: $\mathrm{ML} \pm 2.2$, dorsal/ventral: $\mathrm{DV}$ -5.3 ), mediodorsal thalamus (from Bregma; $10^{\circ}$ angle, AP: $-0.8, \mathrm{ML}: \pm 1.2$, DV: -3.7 ), 
ventral tegmental area (from Bregma; $7^{\circ}$ angle, AP: - 3.2, ML: \pm 1 , DV: -4.6 ), lateral habenula (from Bregma; $10^{\circ}$ angle, AP: $-1.2, \mathrm{ML}: \pm 0.7$, DV: - 3.1).

Double-labeling experiments - Each mouse was injected with two different retrograde tracers, rAAV-GFP and rAAV-RFP (ELSC viral core, The Hebrew University, Israel). Each virus was injected bilaterally ( $300 \mathrm{nl}$ per hemisphere) into one of the VP targets tested here. Two weeks after microinjections mice were anesthetized with Pental (CTS Chemical Industries, Israel) and perfused with 4\% paraformaldehyde (PFA). Sections of the VP $(40 \mu \mathrm{m})$ were prepared using a sliding microtome (Leica, model SM2010R) and mounted on slides. The sections were scanned with Nikon ECLIPSE-NiE fluorescent microscope and scans were analyzed using ImageJ (NIH).

RNA-Sequencing and Bioinformatics- Three weeks following viral injections, VP tissue was collected from Ribotag mice infused with Retrograde-Cre virus (AAV5-Cre).

Projection-specific RNA isolation was performed using polyribosome immunoprecipitation as described previously ${ }^{12,13}$. Briefly, pooled tissue from RiboTag $(R T)+/+$ mice with virally mediated Cre expression in VP projection neurons $(n=4-5$ mice per sample) was homogenized and $800 \mu \mathrm{L}$ of the supernatant was incubated in HAcoupled magnetic beads (Invitrogen: 100.03D; Covance: MMS-101R) overnight at $4^{\circ} \mathrm{C}$. Magnetic beads were washed using high salt buffer. Following TRK lysis buffer, RNA was extracted with the RNeasy Micro kit (Qiagen: 74004). Libraries were prepared from $10 \mathrm{ng}$ of RNA using the NEBNext Ultra kit (New England BioLabs, Ipswich, MA, USA) and sequenced on an Illumina HiSeq 4000 with a 75 bp paired-end read.

Total of 75-110 million reads were obtained for each sample. Reads were aligned to the mouse genome using TopHat and the number of reads that aligned to the predicted coding regions was determined using HTSeq. Significant differential expression was assessed using Limma. Genes with the absolute value of log fold change (LFC) $\geq 0.3$ and an uncorrected $p$-value $<0.05$ in the pairwise comparisons were used for downstream analysis. Cytoscape 3.7.2 software was used for downstream analysis: transcriptional regulator networks were identified using the iRegulon app and Gene Ontology functional enrichment analysis was performed using the BiNGO app. From these lists, top GO terms were selected based off of highest $-\log _{10}$ (adjusted-p-value), and top upstream regulators were selected based on the number of predicted targets DEGs. 
Slice preparation - As described before ${ }^{14-16}$. Mice were anesthetized with $150 \mathrm{mg} / \mathrm{kg}$ Ketamine- $\mathrm{HCl}$ and then decapitated. Coronal slices $(200 \mu \mathrm{m})$ of the VP were prepared (VT1200S Leica vibratome) and moved to vials containing artificial cerebrospinal fluid (aCSF (in mM): $126 \mathrm{NaCl}, 1.4 \mathrm{NaH}_{2} \mathrm{PO}_{4}, 25 \mathrm{NaHCO}_{3}, 11$ glucose, $1.2 \mathrm{MgCl}_{2}, 2.4 \mathrm{CaCl}_{2}$, $2.5 \mathrm{KCl}, 2.0 \mathrm{Na}$ Pyruvate, 0.4 ascorbic acid, bubbled with $95 \% \mathrm{O}_{2}$ and $5 \% \mathrm{CO}_{2}$ ) and a mixture of $5 \mathrm{mM}$ kynurenic acid and $100 \mathrm{mM}$ MK-801. Slices were stored in room temperature $\left(22-24{ }^{\circ} \mathrm{C}\right)$ until recording.

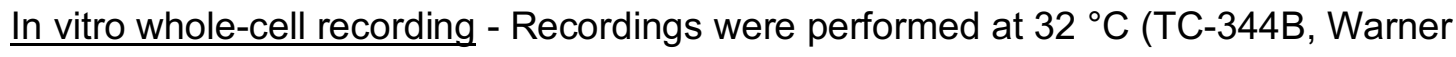
Instrument Corporation). VP neurons were visualized using an Olympus BX51WI microscope and recorded from using glass pipettes (1.3-2 $\mathrm{M} \Omega$, World Precision Instruments) filled with internal solution (in mM: $68 \mathrm{KCl}, 65 \mathrm{D}$-gluconic acid potassium salt, 7.5 HEPES potassium, 1 EGTA, 1.25 MgCl2, $10 \mathrm{NaCl}, 2.0 \mathrm{MgATP}$, and 0.4 NaGTP, pH 7.2-7.3, 275mOsm). Multiclamp 700B (Axon Instruments, Union City, CA) was used to record both membrane and action potentials and inhibitory postsynaptic currents (IPSCs) in whole-cell configuration. Excitability and passive membrane properties were measured in current clamp mode while synaptic activity was measured in voltage clamp mode at a holding potential of $-80 \mathrm{mV}$. Recordings were acquired at 10 $\mathrm{kHz}$ and filtered at $2 \mathrm{kHz}$ using AxoGraph X software (AxoGraph Scientific, Sydney). To evoke IPSCs from NAc terminals expressing ChR2 we used a $470 \mathrm{~nm}$ LED light source (Mightex Systems; $0.1-1 \mathrm{~ms}$ in duration) directed at the slice through the $x 60$ objective. The stimulation intensity was set to evoke $50 \%$ of maximal IPSC at $-80 \mathrm{mV}$. Recordings were collected every 10 seconds. Series resistance (Rs) measured with a $-2 \mathrm{mV}$ hyperpolarizing step (10 ms) given with each stimulus and holding current were always monitored online. Recordings with unstable Rs or when Rs exceeded $20 \mathrm{M} \Omega$ were aborted.

Current clamp experiments - After penetrating the neuron, we switched to current clamp mode and recorded the resting membrane potential of the neurons and spontaneous action potentials for 60 seconds. Cells with unstable membrane potential were discarded. Action potentials were later detected by their waveform using the Axograph software, baselined and analyzed. Potentials of $<20 \mathrm{mV}$ in amplitude were discarded. We then applied the current step protocol - five 500 ms-long depolarization current steps ranging from $0 \mathrm{pA}$ to $+80 \mathrm{pA}$ ( $20 \mathrm{pA}$ intervals) were applied, inter-step interval was 3 seconds. The 5 -step protocol was repeated 5 times with $3 \mathrm{~s}$ between repetitions. 
Baseline membrane potential was adjusted to be approximately $-50 \mathrm{mV}$ in all neurons by injecting constant current.

Cocaine conditioned place preference - Mice were first injected with rAAV-Cre in one of the 4 VP targets examined here and with an AAV encoding for the Gi-coupled DREADD, hM4Di, in a Cre-dependent manner (AAV-DIO-hM4Di-mCherry), or a sham virus (AAV2EF1a-DIO-EYFP-WPRE-pA) into the VP. After two weeks of acclimation to the reverse light cycle and recovery from surgery, mice were trained in the unbiased cocaine conditioned place preference (CPP) paradigm as described previously ${ }^{14}$ (Fig. 9). On the first day, mice were allowed to freely explore both sides of a $30 \mathrm{~cm} \times 30 \mathrm{~cm}$ arena, divided in two, each side with a different wall pattern and floor texture. On the following days, experimental mice received one daily injection of either cocaine (15 mg/kg, i.p.) or saline such that cocaine was always given in one side of the box (the "cocaine-paired" side) and saline in the other. Cocaine-paired sides were counterbalanced for pattern and side. Cocaine/saline injections alternated daily until each mouse received 4 of each. Control mice received 8 injections of saline. Then, mice were left in their cages for 14 days (abstinence) before being tested for their preference of the cocaine-paired side. On the test day, mice first received an injection of the DREADD ligand clozapine-N-oxide (CNO, $3 \mathrm{mg} / \mathrm{kg}$, i.p.), were left in their home cages for 30 minutes and then put in the CPP box for 15 minutes. During the test, mice were allowed to move freely between the two sides of the arena. Movement was tracked using MediaRecorder (Noldus, the Netherlands), analyzed using Optimouse software ${ }^{17}$ and CPP scores were calculated offline as the ratio between the difference in time spent between the cocaine-paired and unpaired sides and the total time [CPP score $=$ (time in paired zone - time in unpaired zone)/(time in paired zone + time in unpaired zone)].

\section{Viruses -}

\begin{tabular}{|l|l|l|l|l|}
\hline Viral construct & manufacturer & $\begin{array}{l}\text { Abbreviation in } \\
\text { paper }\end{array}$ & titer & $\begin{array}{l}\text { Used in } \\
\text { figure \# }\end{array}$ \\
\hline AAVr-hSyn-eGFP & EVCF & rAAV-GFP & $1.78 \mathrm{E}+13$ & 1 \\
\hline AAVr-hSyn-mRuby2 & EVCF & rAAV-RFP & $3.06 \mathrm{E}+13$ & 1 \\
\hline AAVr-hSyn-iCre & EVCF & rAAV-Cre & $1.10 \mathrm{E}+14$ & 8 \\
\hline $\begin{array}{l}\text { AAV2-EF1a-DIO- } \\
\text { hChR2(H134R)-EYFP }\end{array}$ & UNC & AAV-DIO-ChR & $5.7 \mathrm{E}+12$ & 4 \\
\hline
\end{tabular}




\begin{tabular}{|l|l|l|l|l|}
\hline $\begin{array}{l}\text { AAV2-hSyn-DIO- } \\
\text { hM4D(Gi)-mCherry }\end{array}$ & $\begin{array}{l}\text { AAV-DIO- } \\
\text { DREADDs }\end{array}$ & $3.7 \mathrm{E}+12$ & 8 \\
\hline \\
$\begin{array}{l}\text { EVCF - ELSC Vector Core Facility, The Hebrew University of Jerusalem; Salk - Salk Institute } \\
\text { Viral Vector Core; UNC - University of North Carolina Vector Core }\end{array}$ \\
\hline
\end{tabular}

Statistics and data analysis - All statistical analyses were performed using GraphPad Prism 9.2 (GraphPad Software Inc.). Parametric tests were used unless stated otherwise with $p$ values $<0.05$ considered as significant. All tests are indicated in the figure legends. Data in graphs represent mean \pm s.e.m.

\section{$\underline{\text { Results }}$}

\section{Minimal overlap between VP projection neurons}

While the VP projects to the LH, VTA, MDT and LHb, it is not known whether there is any overlap between these projections, and if so to what extent. To examine this we performed six experiments covering all pair combinations of the four targets of the VP. For each pair combination we injected one retrograde virus expressing GFP (rAAV-GFP) into one of the targets and another retrograde virus expressing RFP (rAAV-RFP) into the other target (Fig. 1A). Then we examined the proportion of the double-labeled yellow neurons out of all labeled neurons in the VP (Fig. 1B). Our data show that overlap between projections range between $7-20 \%$, with the $V P_{\rightarrow L H}$ projections showing the lowest overlap proportions and especially low overlap with $V P \rightarrow V T A$ projections $(7 \%)$ (Fig. 1C). This is surprising, as a previous study ${ }^{18}$ showed that most of the VP projection neurons, regardless of their final target, pass through the LH. It is possible that the levels of overlap between VP projections reported here are underestimations as the overlap may be affected by the efficiency of virus uptake by axons and by microinjection localization.

\section{Distinct molecular signatures within VP projection neurons}

After finding that the projection patterns of the VP are relatively distinct to downstream regions, we aimed to examine whether these sets of VP projection neurons exhibit distinct profiles at the molecular level. Although studies have identified the neurochemical composition of VP projection neurons using in situ hybridization ${ }^{3,4,19}$, we profiled global translatome-wide signatures within VP projection neurons. This allows us to identify 
patterns and novel genes enriched within VP projection neurons.To this end, gene expression profiles of VP projection neurons were assessed using RNA-sequencing. Retrograde Cre virus (AAV5-Cre) was infused into one of the following regions in male Ribotag mice: VTA $(n=16), \operatorname{LHb}(n=16), \operatorname{LH}(n=16), \operatorname{MDT}(n=16), \operatorname{VP}(n=16)$. This results in Cre-dependent expression of HA-tagged ribosomal protein within upstream VP neurons. Infusion of AAV5-Cre directly into the VP served as a comparison of non-pathway specific VP (global VP) gene expression. VP tissue was collected from mice and Ribotag methods were used to isolate pathway-specific ribosome-associated mRNA from VP neurons. RNA-Sequencing libraries were constructed from each VP projection group and followed by translatome profiling (Fig. 2A).

First, pair-wise comparisons of gene expression patterns from $V P \rightarrow V T A, V P \rightarrow L H b$, $V P \rightarrow L H$ and $V P \rightarrow M D T$ were compared to gene expression within non-projection specific $V P$ neurons (Fig. 2B). The following number of differentially expressed genes (DEGs) were detected in each VP projection neuron: VP $\rightarrow$ MDT: 4318; VP $\rightarrow$ VTA: 1982; VP $\rightarrow$ LHb:1709 and $V P \rightarrow L H:$ 1308. To further characterize global gene expression patterns, we performed Rank-Rank Hypergeometric Overlap (RRHO) analysis ${ }^{20}$, which compares gene expression between two lists in a threshold free manner. Heat maps display overlap at those points, determined by relative effect sizes in differential gene expression, using log10(p-value). Comparisons between gene expression lists of VP projection neurons

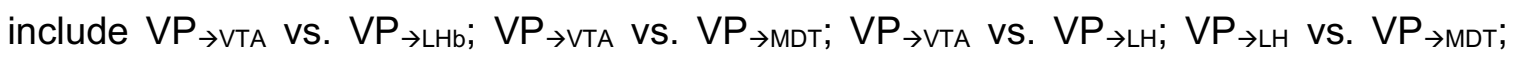
$V P \rightarrow L H b$ Vs. VP $\rightarrow M D T$ and $V P_{\rightarrow L H b}$ Vs. $V P_{\rightarrow L H}$ (Fig. 2D). High concordance is seen within genes upregulated within $V P \rightarrow L H$ versus $V P_{\rightarrow M D T}$ in relation to VP global neurons (peak: $\log 10\left(p\right.$-value): 541) and $V P_{\rightarrow L H b}$ versus $V P_{\rightarrow M D T}$ (peak: -log10(p-value): 334). High

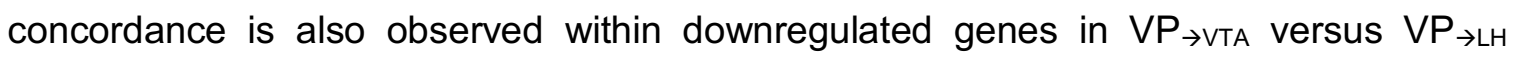
neurons (peak: -log10(p-value): 393). These data overall suggest that VP $\rightarrow$ MDT has the highest number of upregulated genes and exhibits the highest overlap of upregulated gene expression patterns among other VP projection neuron types.

To identify cell-type specific molecules and processes, additional analysis was performed on genes upregulated (i.e. enriched) within the VP neuronal types. VP $\rightarrow$ MDT neurons had the highest number of upregulated DEGs (708) in comparison to other VP

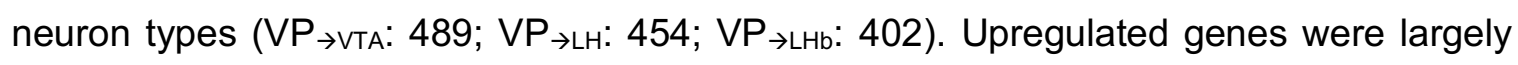
distinct from one another (Fig. 3A), with only 66 genes shared amongst all VP neuron types. Consistent with the patterns detected from RRHO analysis, VP $\rightarrow M D T$ and $V P \rightarrow L H$ 
projection neurons shared the highest number of upregulated genes (267 total) and VP $\rightarrow$ LH and $\mathrm{VP} \rightarrow \mathrm{LHb}$ have little gene overlap (133 total). To characterize the biological processes and cellular functions associated with genes enriched within each VP neuronal type, gene ontology (GO) analysis was performed (Fig. 3B). All projection neuron types have enrichment for GABA receptor activity processes, suggesting that all these neuronal types receive GABAergic inputs. VP $\rightarrow$ VTA neurons have genes enriched for glutamate receptor activity and $V P \rightarrow L H b$ neurons are enriched for dopamine binding, which were not detected in other projection neuron types. Interestingly, shared GO terms across VP projection types include synaptic transmission and cytoskeletal protein binding. This may indicate that VP projection neurons express distinct sets of genes to regulate processes for synaptic communication. Similarly, GO terms for metabolic process and mitochondrian organization are detected amongst $V P_{\rightarrow V T A}$ and $V P_{\rightarrow M D T}$ neurons. This suggests that neuronal types may have distinct molecules that regulate mitochondrial and energyprocesses. Overall, the enriched genes and biological processes within each projection neuron type may underlie the VP projection-specific functions and responses.

\section{The VP $\rightarrow$ MDT projection receives less inhibitory input from the other VP projections}

The projections of the VP may differ not only in their basal gene expression (Fig. 2) but also in the synaptic inputs they receive. To examine this, we used the whole-cell patch clamp technique and recorded from each of the four VP projections the evoked input from the NAc (Fig. 4) and the global spontaneous inputs they receive (Fig. 5).

Examination of the evoked NAc input to each of the VP projections tested here revealed no difference between the projections (Fig. 4). In all projections tested, the proportion of neurons in which we detected evoked NAc input was similar (between 66-77 \%, Fig. 4AB) and the average amplitude of the optogenetically-evoked inhibitory postsynaptic current (olPSC) was similar (Fig. 4C). This was also true for the paired pulse ratio (PPR) and coefficient of variation of the evoked currents (two measures reflecting the probability of release ${ }^{22,23}$ ) (Fig. 4D-E). Lastly, in all VP projections application of picrotoxin completely abolished the NAc oIPSC (Fig. 4F), indicating a GABAergic synapse.

Comparing the characteristics of the spontaneous postsynaptic currents (sPSCs) between the projections revealed that while they do not differ in the amplitude of the SPSCs (Fig. $\mathbf{5 A}$ ), the $V \mathrm{P}_{\rightarrow \mathrm{MDT}}$ projection shows a significantly lower frequency of sPSCs compared to 
the other projections (Fig. 5B) (Kolmogorov-Smirnov tests; $D=0.13, p=0.0001$ compared to $V P \rightarrow$ LHb $; D=0.16, p<0.0001$ compared to $V P \rightarrow L H ; D=0.14, p<0.0001$ compared to VP $\rightarrow V T A$ ). This difference may be due to less GABAergic input because when we washed the slices with picrotoxin $(100 \mu \mathrm{M})$, a GABA-A receptor antagonist, the reduction in the frequency of sPSCs was significantly smaller in VP $\rightarrow$ MDT neurons $(52.8 \pm 33 \%$ reduction) (Fig. 5C-D) [One-way ANOVA main projection effect, $\mathrm{F}_{(3,79)}=4.06$, $\mathrm{p}=0.01$; post-hoc Tukey's multiple comparisons - compared to $\mathrm{VP}_{\rightarrow L H B}\left(79.0 \pm 14.9 \%\right.$ reduction), $p=0.001$, compared to $\mathrm{VP}_{\rightarrow \mathrm{LH}}$ (74.4 $\pm 14.7 \%$ reduction), $p=0.02$, compared to $V P_{\rightarrow V T A}(73.1 \pm 22.5 \%$ reduction), $p=0.03]$.

$V P_{\rightarrow L H}$ neurons are more excitable and $V P_{\rightarrow V T A}$ are less excitable than the average VP neuron

We next tested various properties that contribute to the excitability of the VP projection neurons (Fig. 6A). The resting membrane potential (Fig. 6B) did not differ between VP projections (One-way ANOVA, $F_{(3,103)}=0.49, p=0.69$; VP $\rightarrow M D T=-46.0 \pm 4.4 \mathrm{mV}$; VP $\rightarrow L H b=-$ $\left.45.8 \pm 4.0 \mathrm{mV} ; \mathrm{VP}_{\rightarrow \mathrm{LH}}=-46.6 \pm 5.8 \mathrm{mV} ; \mathrm{VP}_{\rightarrow \mathrm{VTA}}=-47.2 \pm 4.3 \mathrm{mV}\right)$. In contrast, the firing rate at rest (Fig. 6C-D) did differ between projections (One-way ANOVA, $F_{(3,103)}=3.47, p=0.02$ ). In particular, the $V P \rightarrow L H$ neurons had the highest firing rate $(10.9 \pm 8.1 \mathrm{~Hz})$, which was significantly higher than the firing rate of $\mathrm{VP}_{\rightarrow \mathrm{MDT}}(6.1 \pm 4.8 \mathrm{~Hz}$; Post-hoc Holm-Sidak's multiple comparisons test, $p=0.03)$ and $V P \rightarrow V T A$ neurons $(6.5 \pm 5.2 \mathrm{~Hz}$; Post-hoc HolmSidak's multiple comparisons test, $p=0.04$ ).

When we applied a series of depolarizing current steps to the VP projections (Fig. 6E-G), we found that the projections differed both in the number of action potentials they generated per step (Analysis of covariance (ANCOVA), $F_{(3,260)}=1.06, p=0.37$ for slope comparison; $F_{(3,260)}=6.1, p=0.0005$ for elevation comparison) and in the latency to the first action potential (ANCOVA, $\mathrm{F}_{(3,260)}=4.65, \mathrm{p}=0.003$ for slope comparison). Note that the $\mathrm{VP}_{\rightarrow L \mathrm{LH}}$ projections, who had the highest firing rates at rest (Fig. $6 \mathrm{C}$ ), also showed strong firing and quick response when depolarized.

To evaluate the average excitability of the projections we generated an "excitability index". In each of the measured parameters we pooled all cells together and applied z-scores to all cells. Thus, each neuron had four z-scores (one from each experiment) and the excitability index of a neuron is the average of its four z-scores. Comparison of the excitability indexes between VP projections (Fig. 6H) demonstrated that it differed between projections (One-way ANOVA, $F_{(3,146)}=3.21, p=0.03$ ) and that the $V P \rightarrow L H$ and 
$V_{\rightarrow V T A}$ projections had a significantly different excitability index (Post-hoc Tukey's multiple comparisons test, $p=0.02$ ). Moreover, when comparing the excitability indexes to zero, which reflects the average excitability of a VP neuron, the average excitability index of the $V_{\rightarrow L H}$ neurons was significantly higher than zero (One sample t-test, $t_{(36)}=2.18, p=0.035$ ) while that of $V P \rightarrow V T A$ neurons was significantly lower than zero (One sample t-test, $\left.t_{(31)}=2.15, p=0.039\right)$. Thus, our data suggest that $V P \rightarrow L H$ neurons are more excitable than the average VP neurons while $V P_{\rightarrow V T A}$ neurons are less excitable from the average VP neuron.

\section{Genes critical for ion transport enriched within VTA-projecting and LH-projecting VP neurons}

To identify potential molecules underlying neuronal responses seen between VPVTA and VP-LH neurons, we compared genes enriched within both VP projection neuron types. We found 156 genes enriched within both $V P_{\rightarrow L H}$ and $V P \rightarrow V T A$ neurons (Fig. 7A). GO Term analysis illustrated that the 156 genes belonged to processes related to neuron development, synaptic transmission, GABA signaling pathways, chromatin assembly and ion transport (Fig. 7B). This is consistent with previous work identifying VP GABAergic projections to the $\mathrm{LH}$ and VTA ${ }^{4,6}$. It also suggests that despite electrophysiological differences, there may be shared molecules that regulate presynaptic and synaptic processes between $V P \rightarrow L H$ and $V P \rightarrow V T A$ neurons. Given the differences in excitability between these projection neurons, we focused our analysis on genes associated with ion transport, which includes genes encoding subunits of ion channels and transporters. A heat map shows the genes enriched for ion transport within each projection type and their log-fold changes in comparison to global VP neuron gene expression. GABRA5, the GABA-A receptor alpha 5 subunit, is the highest expressing among both projection neuron types. We next performed predicted transcription analysis on the genes associated with ion transport and found RFX3 as a top predictor regulator of several shared ion transport genes. This presents a possible target for shared ion genes within VP $\rightarrow$ VTA and $V P \rightarrow L H$ neurons. To identify genes and biological processes distinct to each neuronal type, GO term analysis was performed on the unshared genes within VP $\rightarrow$ LH $(298$ genes) and VP $\rightarrow$ VTA (333 genes). Interestingly, both sets of genes were associated with processes related to synaptic transmission and ion transport (Fig. 7C,D). Both neuron types have enriched expression of potassium chanels, despite distinct families expressed within each neuronal type (VP-LH: KCNC1 KCNC2; VP-VTA: KCND2, KCNAB1). Predicted transcription factor 
analysis was performed on synaptic genes enriched within $V P_{\rightarrow L H}$ neurons and VP $\rightarrow$ VTA neurons. We found that RNF138 was a top predicted regulator for a subset of VP $\rightarrow$ LH ion transport genes, and DLX4 was a top predicted regulator for a subset of ion transport genes within $V P \rightarrow V T A$ neurons. Altogether, these data demonstrate that $V P \rightarrow V T A$ and $V P \rightarrow L H$ neurons have sets of ion transport genes that are enriched within each projection neuron type and present novel molecular targets to study VP projection-specific neuronal function.

\section{Opposite roles for $V P_{\rightarrow L H}$ and $V P_{\rightarrow V T A}$ projections in cocaine conditioned place preference}

Our data so far indicates different VP projections show different characteristics in many aspects. In particular, the $V P_{\rightarrow L H}$ and $V P \rightarrow V T A$ projections emerge as largely separate neuronal populations (Fig. 1) with contrasting physiological properties (Fig. 6). In light of these differences between the projections, we next tested whether these projections affect differently the motivation to receive drug reward in a cocaine conditioned place preference (CPP) procedure.

To inhibit the activity of a specific projection (e.g. VP $\rightarrow$ LH) we injected a retrograde AAV encoding for Cre recombinase (rAAV-Cre) in the target area to express Cre in the specific VP projection neurons and expressed the inhibitory version of the Designer Receptor Exclusively Activated by Designer Drugs (DREADDs) in a cre-dependent manner in the VP (AAV-DIO-hM4Di-GFP) (control mice were injected with a sham virus, AAV-DIO-GFP) (Fig. 8A). We then trained mice to associate one side of the CPP box with cocaine using the cocaine CPP paradigm (Fig. 8B, see Methods) and then allowed them to undergo abstinence in their home cages for 14 days. Thirty minutes before the CPP test, we injected all mice with $\mathrm{CNO}$ (3 mg/kg, i.p.) and then tested their preference to the cocainepaired side.

Our data show that inhibiting the different VP projections had different effects on the preference of the cocaine paired side (Fig. 8C-H) (One-way ANOVA, $F_{(3,25)}=5.81$, $\mathrm{p}=0.004)$. In particular, the average CPP score when inhibiting the $V P \rightarrow L H$ projection $(0.34 \pm 0.19)$ was significantly lower than the CPP score when inhibiting the VP $\rightarrow V T A$ $(0.63 \pm 0.10$, Tukey's multiple comparisons test $p=0.005)$ or VP $\rightarrow$ MDT $(0.61 \pm 0.15$, Tukey's multiple comparisons test $p=0.01$ ) projections. Lastly, comparison of the CPP scores to 
that of the control group (injected with CNO but expressing the sham virus, CPP score of $0.51)$ revealed that inhibiting the $\mathrm{VP}_{\rightarrow \mathrm{LH}}$ projection significantly reduced the CPP score (One sample $t$-test, $\mathrm{t}_{(7)}=2.54, \mathrm{p}=0.04$ ) while inhibiting the $\mathrm{VP} \rightarrow \mathrm{VTA}$ projection significantly increased the CPP score (One sample t-test, $t_{(7)}=3.42, p=0.01$ ). Inhibiting the different projections did not change differently the overall locomotion of the mice (Fig. 8I). Overall, our data point to different, and maybe opposite roles of $V P \rightarrow L H$ and $V P \rightarrow V T A$ in cocaine preference.

\section{Discussion}

The VP is a structure largely composed of GABAergic neurons and has numerous downstream projection targets. It has been assumed that the cellular composition of the different projections is largely similar, despite the VP's projection-specific roles in behavior. In this manuscript we provide for the first time a thorough examination of four major outputs of the VP and compare directly their genetic profile, input sources, physiological characteristics and roles in cocaine reward behavior. We find that the different VP projections show distinct characteristics in all properties examined. Each projection has distinct sets of enriched genes (Figs. 2-3), exhibits different levels of excitability and inhibitory input (Figs. 4-6) and has differential roles in regulates rewarding cocaine CPP behavior (Fig. 8). In particular, the VP projections to the LH and VTA stand out as being largely distinct cell populations. They show a minimal level of projection overlap (Fig. 2), express different sets of enriched genes (Fig. 7), have different excitability levels (Fig. 6) and show opposite effects on cocaine CPP (Fig. 8).

\section{$\underline{V P}$ heterogeneity}

Our data suggest that these four projection neurons of the VP differ from each other in multiple aspects, potentially suggesting some of these projections contain distinct subtypes of VP neurons. For instance, we found that VP $\rightarrow L H$ neurons have the lowest overlap of output projections with other VP projection neurons, show enrichment of genes critical for axogenesis and GABAergic receptors and are hyperexcitable. Together, this suggests that $V P \rightarrow L H$ neurons are a distinct subgroup of neurons that are potentially poised to propagate information quickly from their inputs. In addition, $V P \rightarrow V T A$ and $V P \rightarrow L H b$ neurons are enriched for biological processes distinct from other VP projection neurons (VP $\rightarrow$ VTA : 
Glutamate receptor activity; $\mathrm{VP}_{\rightarrow \mathrm{LHb} \text { : }}$ dopamine binding activity) suggesting these neuronal types have distinct inputs from other VP projection neurons. However, VP $\rightarrow M D T$ neurons show similar levels of excitability as average (non-projection specific) VP neurons, share the highest overlap of projection with other VP neurons and similarly display large overlap of gene expression patterns with other VP projection neuron types. Surprisingly, although no differences are seen from evoked responses from NAc inputs, VP $\rightarrow$ MDT neurons display lower frequency of of sPSCs from global inputs. Collectively, our viral tracing and molecular profiling suggests that $\mathrm{VP}_{\rightarrow \mathrm{MDT}}$ neurons may have overlapping cells with other VP projections, however our electrophysiological data suggests they differ in their inhibitory inputs with other VP neurons. These $V P_{\rightarrow M D T}$ neurons, although they receive inhibitiory inputs from NAc, may receive information from other upstream regions such as the glutamtergic prefrontal cortex or the dopaminergic VTA ${ }^{2}$. We also found no differences in responses from evoked inputs from the NAc among VP projection neurons, which may suggest that these VP neurons do not exhibit baseline differences in responses to GABAergic NAc inputs. Our GO term analysis could reflect this, as all VP projection neurons have genes enriched for GABA receptor activity. However, this may change following responses to other stimuli such as cocaine, as we have previously observed synaptic adaptations in VP projection neurons during cocaine abstinence ${ }^{15}$. Our data overall suggests that VP projection neurons, with the exception of VP $\rightarrow$ MDT neurons, are largely non-overlapping and composed of distinct genetic and cellular profiles. These findings also highlight the need to further interrogate how the VP encodes distinct sets of information for its various inputs to promote stimuli and input-specific behaviors.

Although our findings suggest segregation of the VP projection neurons, it is unclear from this data alone how distinct these VP neurons are. Despite the fact that we show low percentage of VP neurons projecting to two targets and that this is corroborated by differences in our other biological measurements, it should be emphasized that they may be underestimates. This is due to the fact that differences in viral expression of retrograde tracers depends on the efficiency of viral uptake and the excact location of the injection. In addition, combining the retrograde tracing data from all projections poses the possibility that a significant proportion of neurons projecting to one target projects also to another target. Thus, while we show that the projections of the VP show distinct characteristics, they may still show overlap with others.

The difference between $V P_{\rightarrow L H}$ and $V P_{\rightarrow V T A}$ projections 
Our results highlight the $\mathrm{VP} \rightarrow \mathrm{LH}$ and the $\mathrm{VP} \rightarrow \mathrm{VTA}$ projections as being particularly distinct from each other. They show only $7 \%$ overlap (Fig. 1) and a large number of upregulated genes within these populations are non-overlapping (Fig. 7). We also detected differences in excitability, with $V P_{\rightarrow L H}$ neurons being more excitable while $V P \rightarrow V T A$ neurons are less excitable than other VP neurons (Fig. 6). Consistent with this, chemogenetic inhibition of these populations resulted in opposite behavioral effects; inhibition of VP $\rightarrow L H$ neurons decreases while inhibition of VP $\rightarrow$ VTA neurons increases cocaine CPP.

The differences we report on $V P \rightarrow L H$ and $V P \rightarrow V T A$ neurons challenge several previous notions of the VP. First, an anatomical study suggests that approximately $90 \%$ of VP projections, including those to the VTA, pass through the $\mathrm{LH}^{18}$. In fact, the $\mathrm{LH}$ is positioned between the VP and the VTA ${ }^{24}$. Our data suggests that these VP projections to the VTA do not collateralize to the $\mathrm{LH}$, and are strictly passing through the structure. Our CPP work does demonstrate that the VP exerts projection-specific effects on behavior, as previously seen ${ }^{3,4,8,25}$. However, there is conflicting reports on the effects of inhibiting VP projection neurons on drug seeking. These differences may be due to different cell-type specific manipulations, as Prasad et al. had found that inhibition of $V P_{\rightarrow L H}$ GABAergic neurons, but not $V P \rightarrow L H$ parvalbumin neurons reduces renewal of operant-based alcohol seeking in rats $^{6}$. This may also be due to species-specific effects, as inhibition of VP $\rightarrow$ VTA neurons has previously been shown to enhance cocaine seeking in mice ${ }^{8}$, but impair cocaine-seeking in rats ${ }^{26}$. Alternatively, it is possible that different circuits and cell-types are affected due to extinction training ${ }^{6,26}$ and forced abstinence ${ }^{8}$. Further investigation into these underlying differences will be required to better understand the role of VP in drug-seeking.

The mechanism allowing the $V P_{\rightarrow L H}$ and the $V P \rightarrow V T A$ projections to have different behavioral roles in drug seeking is still not understood. One possibility is potential opposite effects on dopamine release from VTA neurons. The VP projection to the VTA targets both GABA and dopamine neurons ${ }^{15,27,28}$. It is still not known how activation of VP input to the VTA affects dopamine levels but it is plausible that it enhances dopamine release as it increases the firing rate of putative dopamine neurons ${ }^{26}$. LH GABAergic neurons that project to the VTA increase firing of VTA dopamine neurons ${ }^{29}$. These neurons are also a main target of $V P \rightarrow L H$ projections ${ }^{30}$. Thus, activation of $V P \rightarrow L H$ neurons, which are mostly GABAergic $^{1}$ (Fig. 7) may result in a net decrease in dopamine release. Therefore, VP $\rightarrow V T A$ and $V P \rightarrow L H$ are expected to have opposite effects on dopamine release from the VTA. This may underlie their opposite effect on cocaine CPP. 
Altogether, our data demonstrates that VP projection neurons have relatively distinct pathways, cellular properties and molecular compositions. These baseline differences may reflect their projection-specific roles of cocaine-related behaviors. Our behavioral findings illustrate how VP projection neurons have differential roles of in cocaine reward, which is consistent with previous work in the field. This work emphasizes the importance in further investigating and characterizing the VP projections and cell types in addiction processes.

\section{Bibliography}

1. Kupchik, Y. M. \& Prasad, A. A. Ventral pallidum cellular and pathway specificity in drug seeking. Neurosci. Biobehav. Rev. 131, 373-386 (2021).

2. Root, D. H., Melendez, R. I., Zaborszky, L. \& Napier, T. C. The ventral pallidum: Subregion-specific functional anatomy and roles in motivated behaviors. Prog. Neurobiol. 130, 29-70 (2015).

3. Tooley, J. et al. Glutamatergic Ventral Pallidal Neurons Modulate Activity of the Habenula-Tegmental Circuitry and Constrain Reward Seeking. Biol. Psychiatry 83, 1012-1023 (2018).

4. Faget, L. et al. Opponent control of behavioral reinforcement by inhibitory and excitatory projections from the ventral pallidum. Nat. Commun. 9, 849 (2018).

5. Floresco, S. B., Braaksma, D. N. \& Phillips, A. G. Involvement of the ventral pallidum in working memory tasks with or without a delay. Ann. N. Y. Acad. Sci. 877, 711-716 (1999).

6. Prasad, A. A. et al. Complementary roles for ventral pallidum cell types and their projections in relapse. J. Neurosci. 40, 880-893 (2020).

7. Heinsbroek, J. A. et al. Opposing regulation of cocaine seeking by glutamate and GABA neurons in the ventral pallidum. Cell Rep. 30, 2018-2027.e3 (2020).

8. Pribiag, $\mathrm{H}$. et al. Ventral pallidum DRD3 potentiates a pallido-habenular circuit driving accumbal dopamine release and cocaine seeking. Neuron 109, 21652182.e10 (2021).

9. McKenna, J. T. et al. Distribution and intrinsic membrane properties of basal forebrain GABAergic and parvalbumin neurons in the mouse. J. Comp. Neurol. 521, 1225-1250 (2013).

10. Zaborszky, L., Buhl, D. L., Pobalashingham, S., Bjaalie, J. G. \& Nadasdy, Z. Threedimensional chemoarchitecture of the basal forebrain: spatially specific association 
of cholinergic and calcium binding protein-containing neurons. Neuroscience 136, 697-713 (2005).

11. Schwarz, L. A. et al. Viral-genetic tracing of the input-output organization of a central noradrenaline circuit. Nature 524, 88-92 (2015).

12. Engeln, M. et al. Individual differences in stereotypy and neuron subtype translatome with TrkB deletion. Mol. Psychiatry 26, 1846-1859 (2021).

13. Chandra, R. et al. Opposing role for Egr3 in nucleus accumbens cell subtypes in cocaine action. J. Neurosci. 35, 7927-7937 (2015).

14. Inbar, K. et al. Cocaine Dysregulates Dynorphin Modulation of Inhibitory Neurotransmission in the Ventral Pallidum in a Cell-Type-Specific Manner. J. Neurosci. 40, 1321-1331 (2020).

15. Levi, L. A. et al. Projection-Specific Potentiation of Ventral Pallidal Glutamatergic Outputs after Abstinence from Cocaine. J. Neurosci. 40, 1276-1285 (2020).

16. Gendelis, S., Inbar, D., Inbar, K., Mesner, S. \& Kupchik, Y. M. Metaplasticity in the Ventral Pallidum as a Potential Marker for the Propensity to Gain Weight in Chronic High-Calorie Diet. J. Neurosci. 40, 9725-9735 (2020).

17. Ben-Shaul, Y. OptiMouse: a comprehensive open source program for reliable detection and analysis of mouse body and nose positions. BMC Biol. 15, 41 (2017).

18. Tripathi, A., Prensa, L. \& Mengual, E. Axonal branching patterns of ventral pallidal neurons in the rat. Brain Struct. Funct. 218, 1133-1157 (2013).

19. Vachez, Y. M. et al. Ventral arkypallidal neurons inhibit accumbal firing to promote reward consumption. Nat. Neurosci. 24, 379-390 (2021).

20. Plaisier, S. B., Taschereau, R., Wong, J. A. \& Graeber, T. G. Rank-rank hypergeometric overlap: identification of statistically significant overlap between gene-expression signatures. Nucleic Acids Res. 38, e169 (2010).

21. Osakada, F. \& Callaway, E. M. Design and generation of recombinant rabies virus vectors. Nat. Protoc. 8, 1583-1601 (2013).

22. Berninger, B., Schinder, A. F. \& Poo, M. M. Synaptic reliability correlates with reduced susceptibility to synaptic potentiation by brain-derived neurotrophic factor. Learn. Mem. 6, 232-242 (1999).

23. Schinder, A. F., Berninger, B. \& Poo, M. Postsynaptic target specificity of neurotrophin-induced presynaptic potentiation. Neuron 25, 151-163 (2000).

24. Paxinos, G. \& Franklin, K. B. J. The Mouse Brain in Stereotaxic Coordinates. (Academic Press, 2001).

25. Prasad, A. A. \& McNally, G. P. Ventral Pallidum Output Pathways in ContextInduced Reinstatement of Alcohol Seeking. J. Neurosci. 36, 11716-11726 (2016).

26. Mahler, S. V. et al. Designer receptors show role for ventral pallidum input to ventral tegmental area in cocaine seeking. Nat. Neurosci. 17, 577-585 (2014). 
27. Hjelmstad, G. O., Xia, Y., Margolis, E. B. \& Fields, H. L. Opioid modulation of ventral pallidal afferents to ventral tegmental area neurons. J. Neurosci. 33, 6454-6459 (2013).

28. Watabe-Uchida, M., Zhu, L., Ogawa, S. K., Vamanrao, A. \& Uchida, N. Whole-brain mapping of direct inputs to midbrain dopamine neurons. Neuron 74, 858-873 (2012).

29. Nieh, E. H. et al. Inhibitory Input from the Lateral Hypothalamus to the Ventral Tegmental Area Disinhibits Dopamine Neurons and Promotes Behavioral Activation. Neuron 90, 1286-1298 (2016).

30. Jennings, J. H., Rizzi, G., Stamatakis, A. M., Ung, R. L. \& Stuber, G. D. The inhibitory circuit architecture of the lateral hypothalamus orchestrates feeding. Science 341, 1517-1521 (2013).

\section{Figure legends}

Figure 1 - Minimal overlap between ventral pallidal projections to the MDT, LHb, LH and VTA. (A) Experimental setup. We injected each mouse with two retrograde viruses expressing either RFP (rAAV-RFP) or GFP (rAAV-GFP), each in a different target of the VP. Thus, we could identify neurons projecting to either target and those projecting to both. (B) Sample images of labeled VP $\rightarrow$ MDT neurons, VP $\rightarrow$ LH neurons and double-labeled neurons projecting to both targets. (C) Pie charts depicting for each pair of VP targets the proportion of double-labeled VP neurons projecting to both targets out of all labeled neurons (arranged from lowest to highest proportion of double-labeled neurons). The proportion of VP neurons projecting to both targets tested was in the range of $7-20 \%$. Note that $V P \rightarrow L H$ neurons showed the lowest proportions of overlap, especially with $V P \rightarrow V T A$ neurons.

Figure 2- VP Projection Neurons have distinct molecular signatures. (A) Retrograde Cre (AAV5-Cre) was infused into 1 of 5 downstream VP regions (VTA; LHb; LH; MDT; VP) within Ribotag male mice. HA-immunoprecipitation procedures was employed to isolate RNA from distinct VP projection neurons. RNA-sequencing libraries were generated and analyzed form these samples to characterize baseline gene expression profiles within VP neurons projecting to the VTA, LHb, LH, MDT. (B) Differential Gene Expression (DEG) Analysis was generated by comparing gene expression within specific VP- projection neuron types relative to the global VP gene expression (uncorrected $p<0.05$ ). Transcriptional patterns within each VP projection neuron type are shown in a heat map. (C) Rank-Rank Hypergeometric Overlap (RRHO) Analysis comparing gene expression in a threshold free manner suggests VP-MDT and VP-LH and VP-MDT and VP-LHb projection neurons have the highest concordance of gene expression patterns. 
Figure 3- Gene ontology analysis identifies biological processes and molecular functions enriched within VP projection neurons.

(A) Genes upregulated within each VP projection neuron type were compared in Venn Diagrams. The VP-MDT projection neuron has the highest number of enriched genes, with VP-MDT and VP-LH sharing the highest number of enriched genes. (B) Top Gene Ontology (GO) terms enriched in upregulated genes from each VP projection neuron.

Figure 4 - Similar nucleus accumbens input to different VP projections. (A) Schematic representation of the experimental setup. In each mouse, a retrograde tracer was injected into one of the four targets of the VP examined here and ChR2 was expressed in NAc neurons. Recordings were performed from identified VP projections and NAc input was induced optogenetically. (B) The proportion of responding VP neurons (i.e. the number of neurons displaying an oIPSC divided by the total number of neurons examined) was similar between projections and was between 66-77\%. (C-E) The amplitude (C), paired-pulse ratio (D) and coefficient of variation (E) of the evoked olPSC did not differ between projections (One-way ANOVA; $F_{(3,80)}=0.64$, $p=0.59$ for amplitude; $F_{(3,80)}=1.75, p=0.16$ for paired-pulse ratio; $F_{(3,80)}=1.08, p=0.36$ for $\mathrm{CV}$ of amplitude). (F) Application of $100 \mu \mathrm{M}$ picrotoxin (GABA-A receptor blocker) on the slice completely blocked the oIPSCs in all VP projections, indicating these are GABA-only inputs. Inset - representative traces.

Figure 5 - Projection-specific differences in the overall inhibitory input to VP neurons. (A) Cumulative probability of SPSC amplitudes in the four VP projections. There were no differences between the curves (Kolmogorov-Smirnov tests; $p>0.16$ for all pairwise comparisons between projections). (B) Cumulative probability of the frequency of sPSCs in the four VP projections. The VP $\rightarrow$ MDT neurons showed significantly lower frequencies compared to the other projections (Kolmogorov-Smirnov tests; $d=0.13$, $p=0.0001$ compared to VP $\rightarrow\llcorner H b ; d=0.16, p<0.0001$ compared to VP $\rightarrow$ LH; $d=0.14, p<0.0001$ compared to VP $\rightarrow$ VTA). * $-p<0.05$ for MDT compared with each of the other projections. (C-D) Application of $100 \mu \mathrm{M}$ picrotoxin (GABA-A receptor blocker) on the slice significantly reduced the frequency of spontaneous inputs in all projections (C), but the reduction was smaller for the $\mathrm{VP}_{\rightarrow \mathrm{MDT}}$ projections (D) (frequency was reduced to $47.2 \pm 7$ $\%$ for $\mathrm{VP}_{\rightarrow \mathrm{MDT}}, 21.1 \pm 3 \%$ for $\mathrm{VP} \rightarrow \mathrm{LHb}, 25.7 \pm 3 \%$ for $\mathrm{VP} \rightarrow \mathrm{LH}$ and $26.9 \pm 5 \%$ for $\mathrm{VP} \rightarrow \mathrm{VTA}$ neurons; * $-p<0.05$ compared to $V P \rightarrow M D T$ neurons).

Figure 6 - VP $\rightarrow$ LH neurons are more excitable and VP $\rightarrow$ VTA neurons are less excitable than the average VP neuron. (A) Experimental setup. VP projections were labeled by injecting a retrograde tracer in the target region and recorded from using whole-cell patch clamp electrophysiology. (B) Resting membrane potential was not different between the four VP projections we examined (One-way ANOVA, $F_{(3,103)}=0.49$, $\mathrm{p}=0.69$; VP $\rightarrow$ MDT $=-46.0 \pm 4.4 \mathrm{mV} ; \mathrm{VP}_{\rightarrow \mathrm{LHb}}=-45.8 \pm 4.0 \mathrm{mV} ; \mathrm{VP} \rightarrow \mathrm{LH}=-46.6 \pm 5.8 \mathrm{mV} ; \mathrm{VP} \rightarrow \mathrm{VTA}=-$ $47.2 \pm 4.3 \mathrm{mV}$ ). (C) Action potential firing frequency at rest differed between the different VP projections (One-way ANOVA, $F_{(3,103)}=3.47$, $p=0.02$ ). Post-hoc analyses show that 
$V_{\rightarrow L H}$ neurons fire in higher frequencies than $\mathrm{VP}_{\rightarrow \mathrm{MDT}}$ (Post-hoc Holm-Sidak's multiple comparisons test, $p=0.03$ ) and VP $\rightarrow$ VTA (Post-hoc Holm-Sidak's multiple comparisons test, $p=0.04$ ) neurons. (D) Representative traces. (E-F) When applying a series of increasing depolarizing steps (inset, 20,40,60 and $80 \mathrm{pA}$ ) we found a main projection effect both in the number of action potentials per step (Analysis of covariance (ANCOVA), $F_{(3,260)}=1.06, p=0.37$ for slope comparison; $F_{(3,260)}=6.1, p=0.0005$ for elevation comparison) (E) and in the minimal latency to fire the first action potential (ANCOVA, $F_{(3,260)}=4.65, p=0.003$ for slope comparison) $(F)$. Note that $V P \rightarrow L H$ neurons had among the highest number of action potentials and shortest latency to fire, both of which indicate high excitability. (G) Representative traces. (H) An "Excitability index" was calculated by first calculating for each cell a z-score in each of the parameters recorded in panels $B, C, E, F$ and then calculating the average $z$-score for each cell. There was a main projection effect for the $z$-scores (One-way ANOVA, $F_{(3,146)}=3.21, p=0.03$ ) and posthoc analyses (Tukey's multiple comparisons test) revealed that the $\mathrm{VP} \rightarrow \mathrm{LH}$ and the $V P \rightarrow$ VTA projections were significantly different in their excitability $(p=0.02)$. In addition, the excitability index of $V P_{\rightarrow L H}$ neurons was significantly higher than zero (One sample $t$ test, $\left.\mathrm{t}_{(36)}=2.18, \mathrm{p}=0.035\right)$ while that of $\mathrm{VP} \rightarrow \mathrm{VTA}$ neurons was significantly lower than zero (One sample t-test, $t_{(31)}=2.15, p=0.039$ ). Thus, $V P \rightarrow L H$ neurons are more excitable and $V P \rightarrow$ VTA neurons are less excitable than the average VP neuron. ${ }^{*}-p<0.05$ compared to zero.

Figure 7 - Distinct sets of genes related to ion transport are enriched within VTAprojecting and LH-projecting VP neurons. (A) Upregulated genes within VP $\rightarrow L H$ and $V P \rightarrow$ VTA projection neurons were compared in a Venn diagram. Gene Ontology analysis identifies common synaptic genes $(B)$ in VP $\rightarrow V T A$ and $V P \rightarrow L H$ projection neurons (log fold changes from each projection type displayed in a heat map) and RFX3 as a predicted upstream regulator of a subset of genes related to ion transport. GO term analysis reveals distinct sets of ion transport related genes and their listed potential upstream regulators within the $\mathrm{VP}_{\rightarrow \mathrm{LH}}(\mathbf{C})$ and $\mathrm{VP} \rightarrow \mathrm{VTA}$ projection neurons (D).

Figure 8 - Inhibiting VP $\rightarrow$ LH neurons diminishes, while inhibiting VP $\rightarrow$ VTA neurons enhances the preference for cocaine. (A) Viral injection strategy. A retrograde AAV expressing Cre (rAAV-Cre) was injected into one of the 4 targets of the VP tested here and an AAV encoding Cre-dependently for the inhibitory DREADD hM4Di (AAV-DIOhM4Di-eYFP) or a control sham virus (AAV-DIO-eYFP) were injected into the VP. (B) Cocaine CPP paradigm. After habituating to the CPP box, mice received daily alternating injections of cocaine (15 mg/kg i.p.) in one side of the box or saline in the other side of the box (4 injections of each). Mice then underwent 14 days of abstinence in their home-cages. On the test day, all mice first received an injection of CNO $(3 \mathrm{mg} / \mathrm{kg}$ i.p.) 30 minutes prior to the beginning of the test and then were left in the CPP box for 15 minutes, during which their movement was recorded. A CPP score was calculated using the following equation - CPP score $=\frac{\text { Time in cocaine }- \text { paired side }- \text { Time in saline-paired side }}{\text { Total time in the CPP box }}$. (C) Inhibiting different VP projections had different influences on behavior (One-way ANOVA, $\left.F_{(3,25)}=5.81, p=0.004\right)$. While inhibiting $V P_{\rightarrow M D T}$ or $V P \rightarrow L H b$ neurons did not affect the CPP score (One-sample t-test comparing to the CPP score of control mice expressing the sham virus and receiving $C N O$, Sham $X C N O, 0.51 ; t_{(6)}=1.71, p=0.14$ for $\mathrm{VP}_{\rightarrow \mathrm{MDT}}, \mathrm{t}_{(5)}=0.38, \mathrm{p}=0.72$ for $\left.\mathrm{VP} \rightarrow \mathrm{LHb}\right)$, inhibiting the $\mathrm{VP}_{\rightarrow \mathrm{LH}}$ projection decreased the CPP 
score $\left(\mathrm{t}_{(7)}=2.54, \mathrm{p}=0.039\right)$ and inhibiting the $\mathrm{VP} \rightarrow \mathrm{VTA}$ projection increased the CPP score $\left(t_{(7)}=3.42, p=0.011\right)$. (D-H) Heatmaps of the movement of representative mice from all groups. (I) Inhibiting the different VP projections did not affect the distance traveled differently between projections. 


\section{Figure 1}
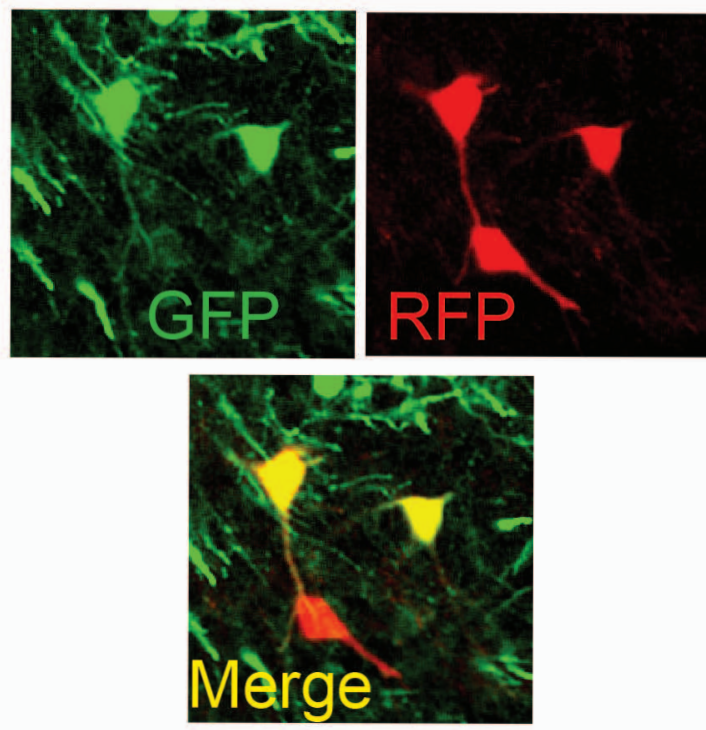

C

$7 \%$

rAAV-GFP

(14/208)
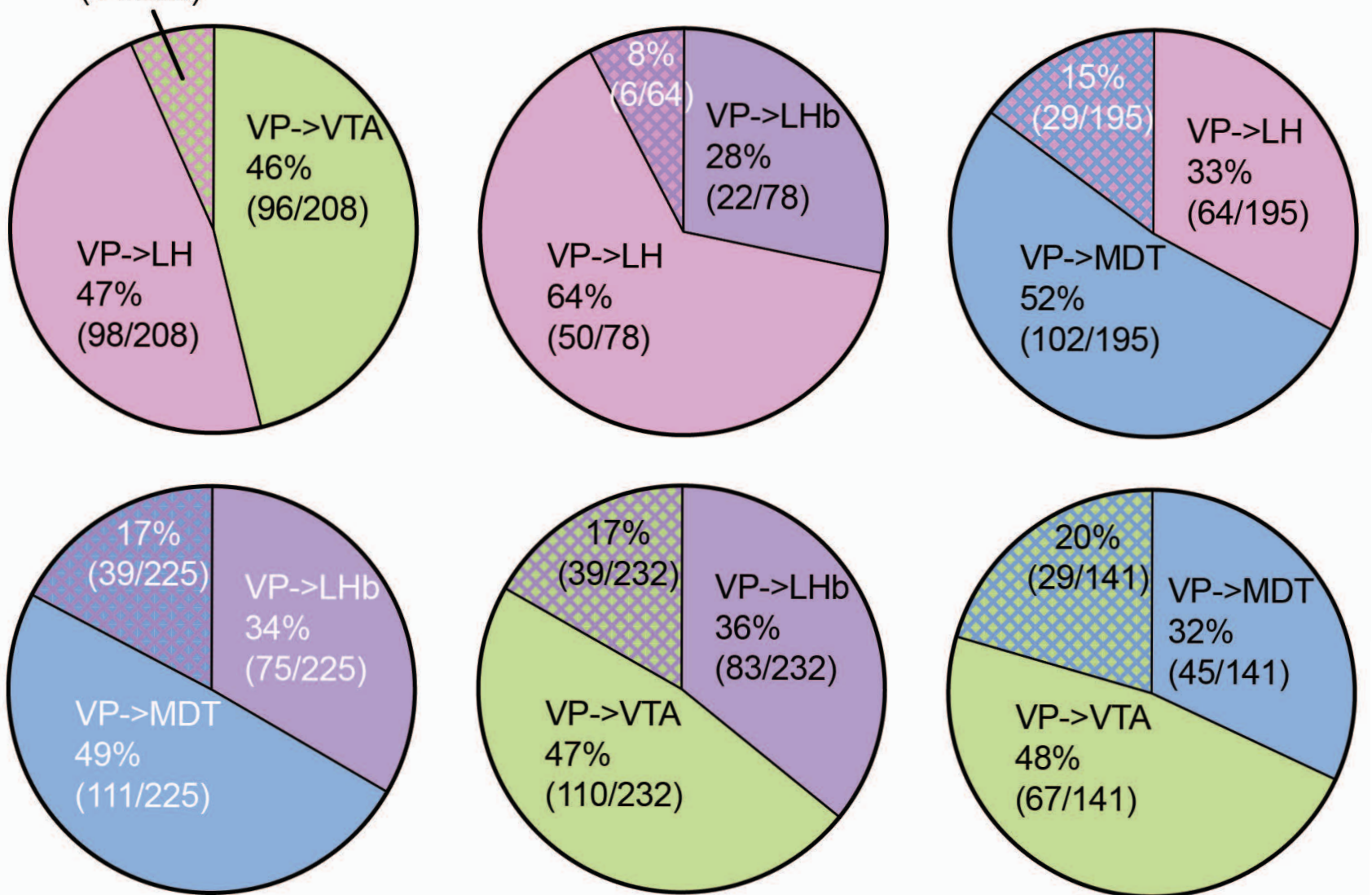


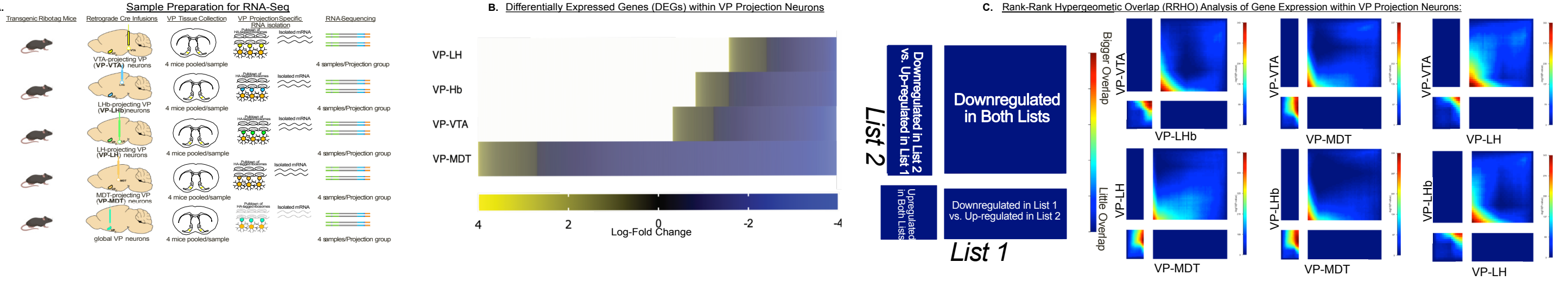

Figure 2 
B. Gene Ontology Analysis of Upregulated DEGs within VP-Projection Neurons

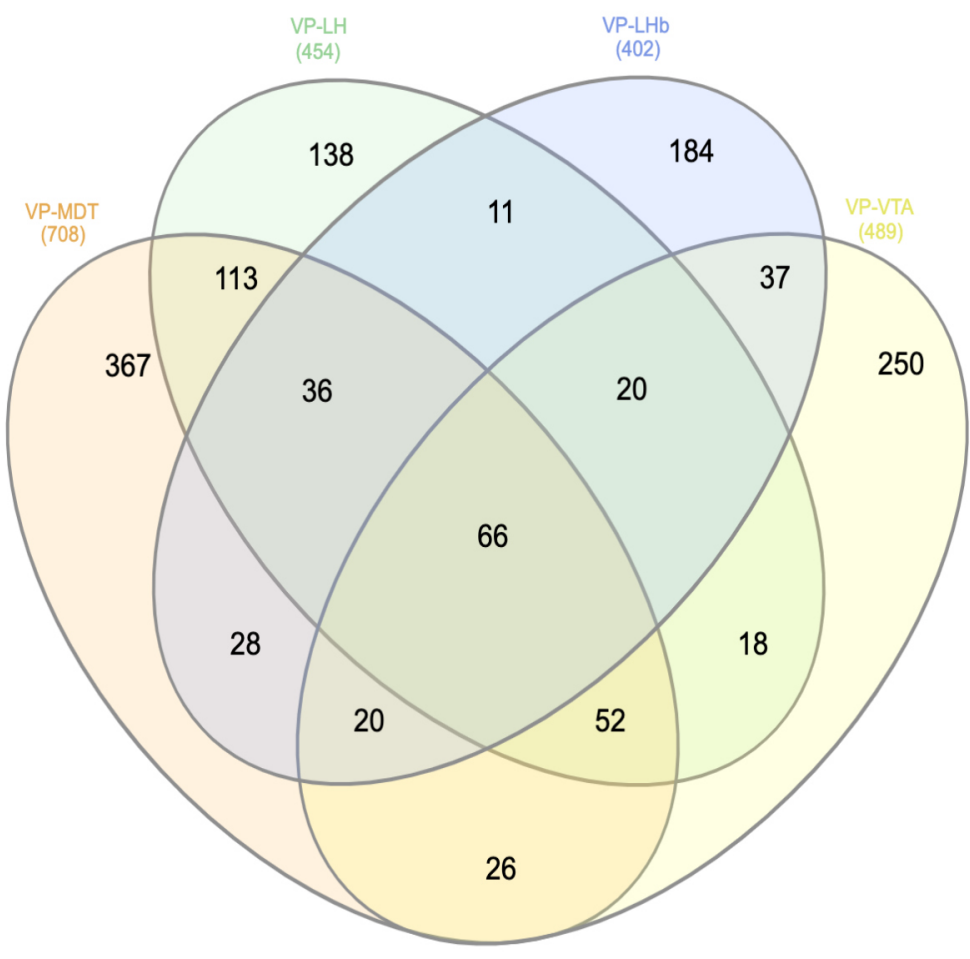

Figure 3

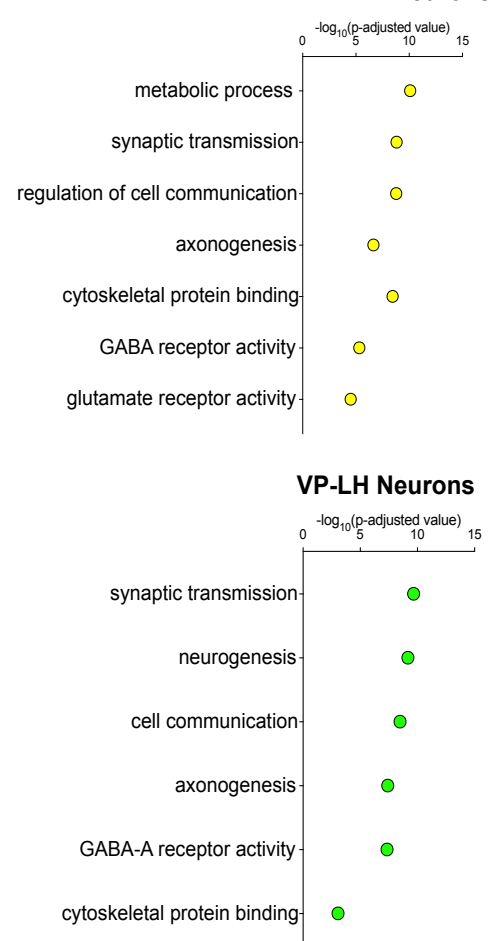

VP-VTA Neurons

cytoskeletal protein binding

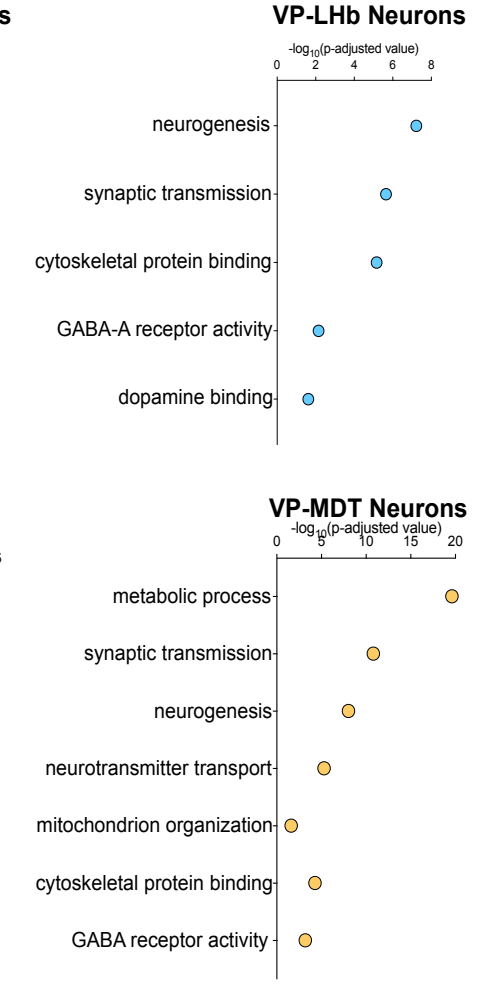


A Figure 4
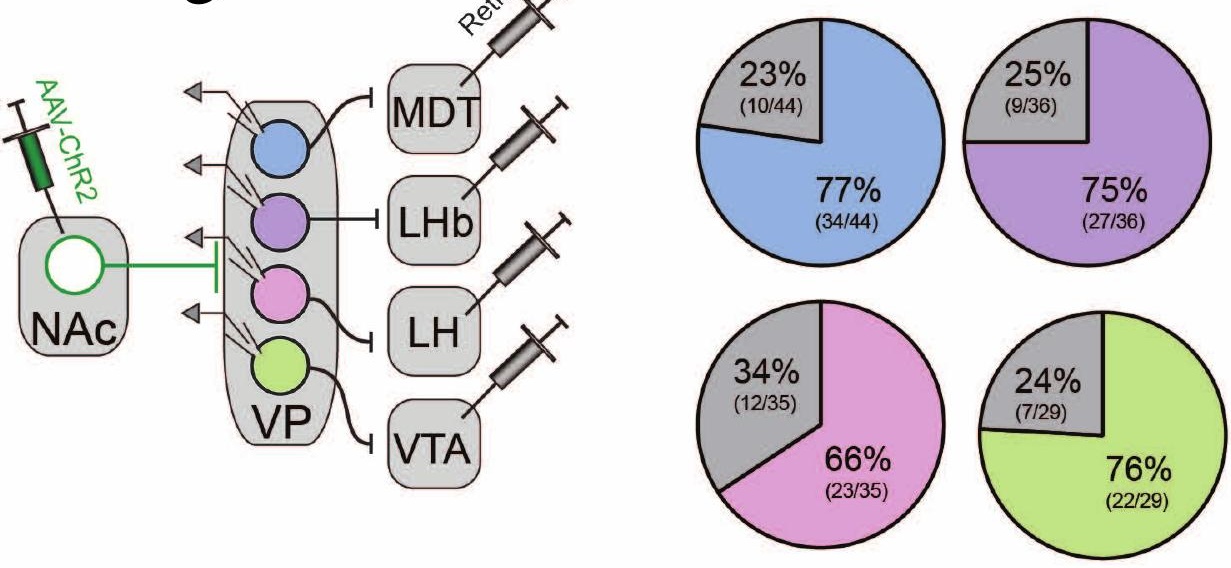

0

D
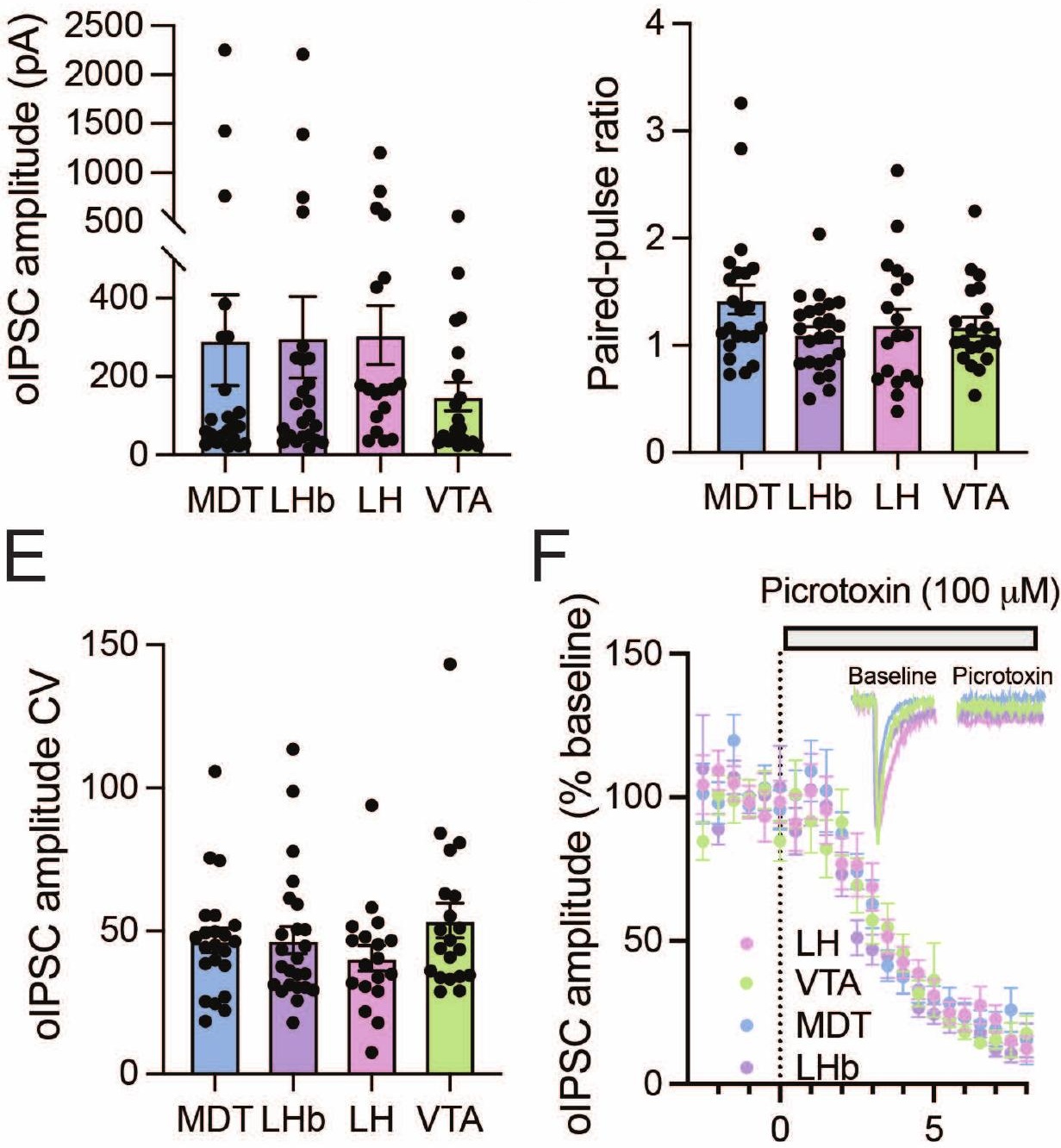

Time (Min) 
A Figure 5

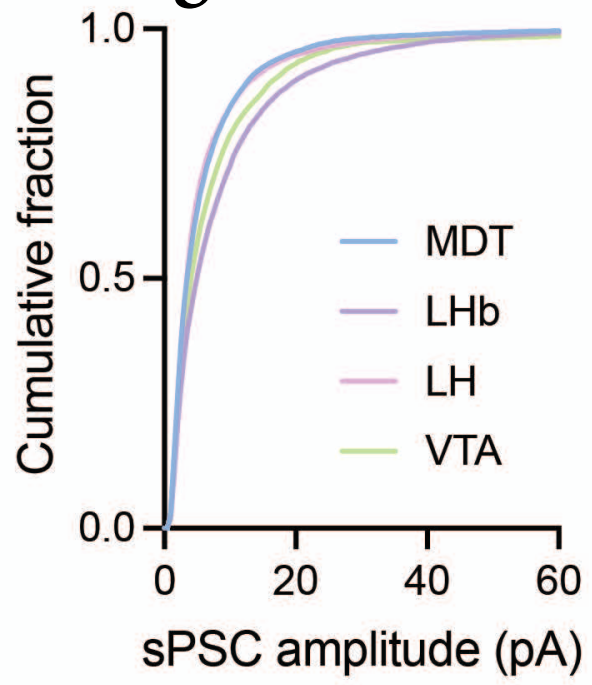

B

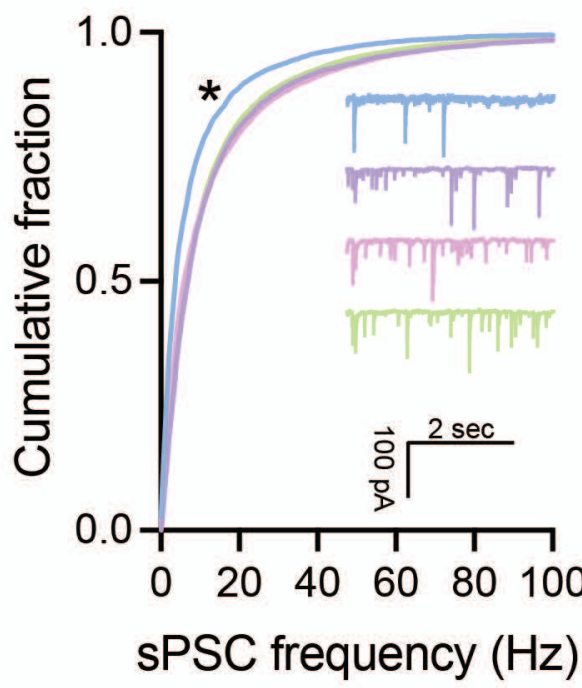

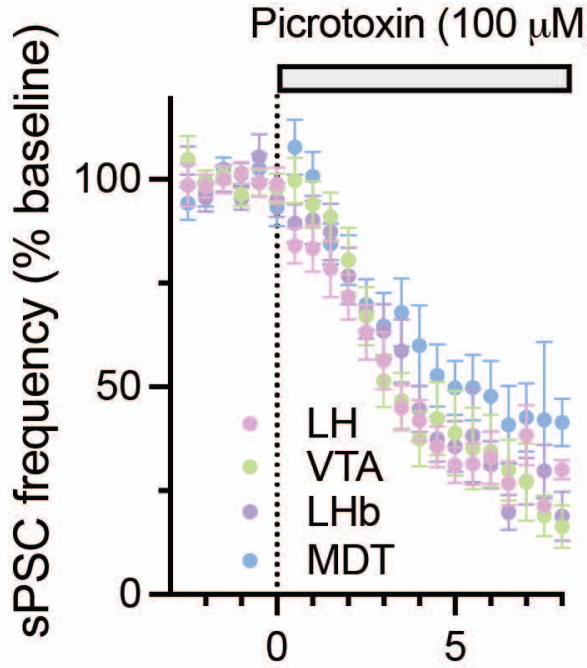

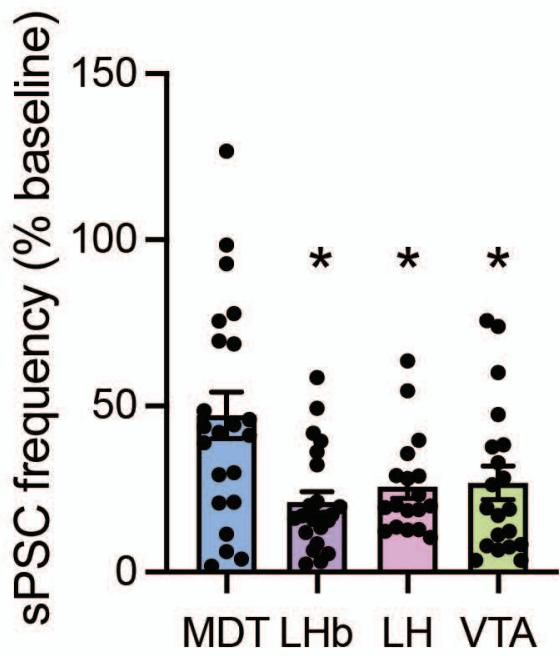

Time (Min) 
Figure 7

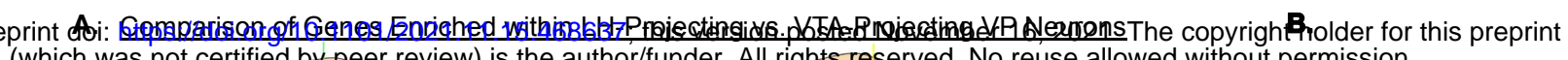

Bolder for th
permission.

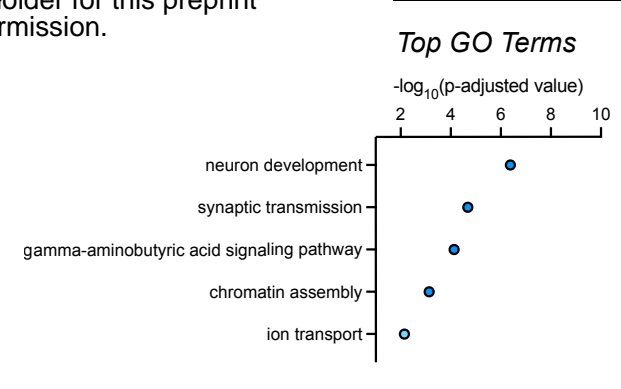

Enriched Genes Specific to LH-projecting VP Neurons vs. VTA Projecting VP Neurons

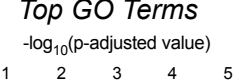
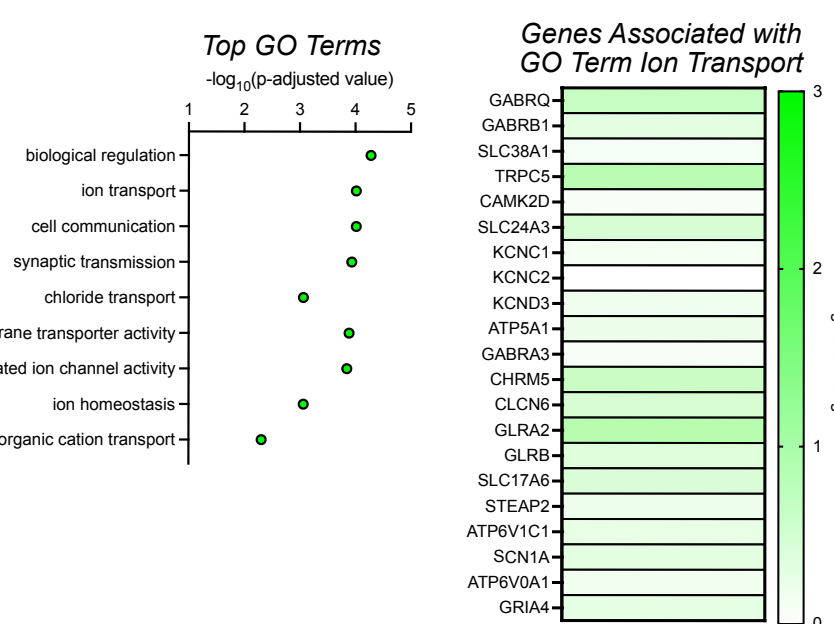

Predicted Transcription Factor Regulating VP-LH
Ion Transport Genes

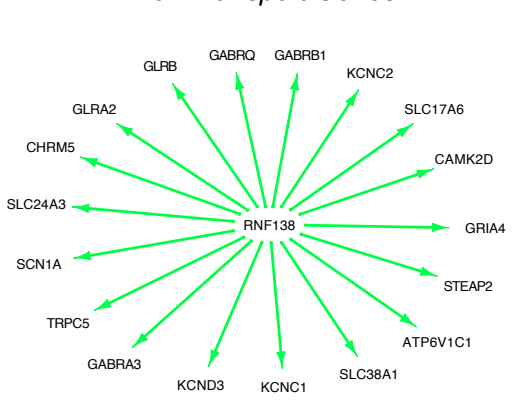

D.

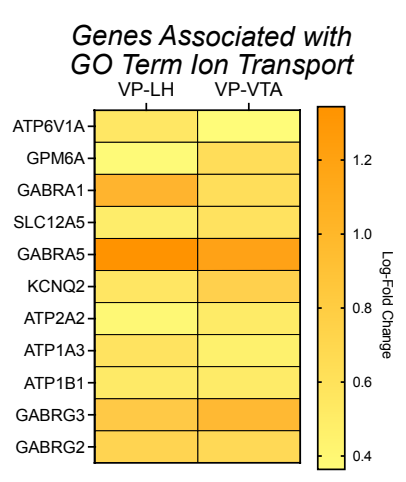

Predicted Transcription Factor
Regulating VP-LH and VP-VTA Regulating VP-LH and VP-VTA
Ion Transport Genes

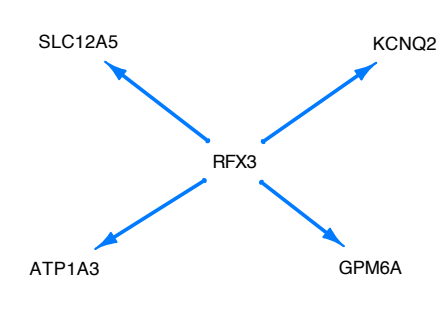

Enriched Genes Specific to VTA-projecting VP Neurons vs. LH-Projecting VP Neurons
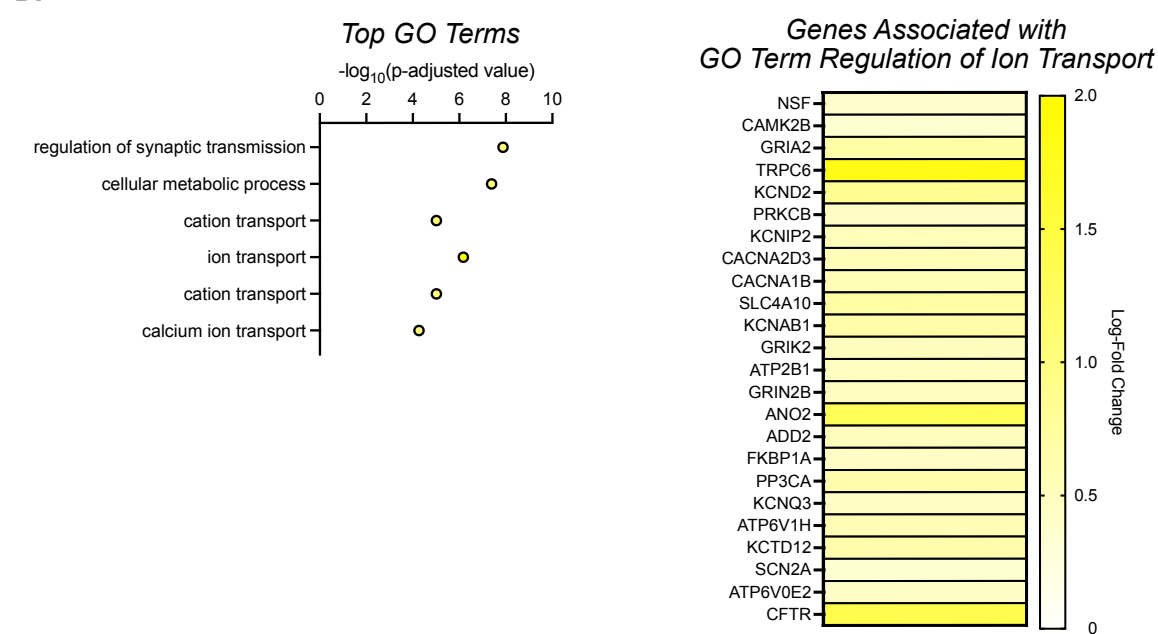

Predicted Transcription Factor

Pedicted Transcription Factor
Regulating VP-VTA
lon Transport Genes

GRIN2B CACNA2D3

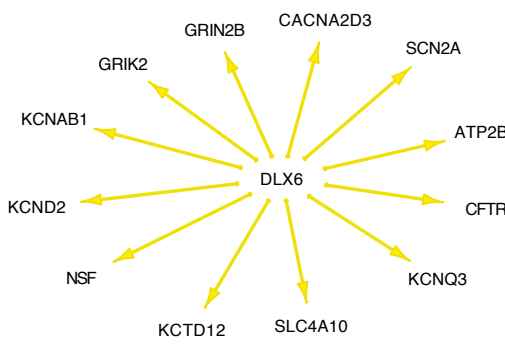




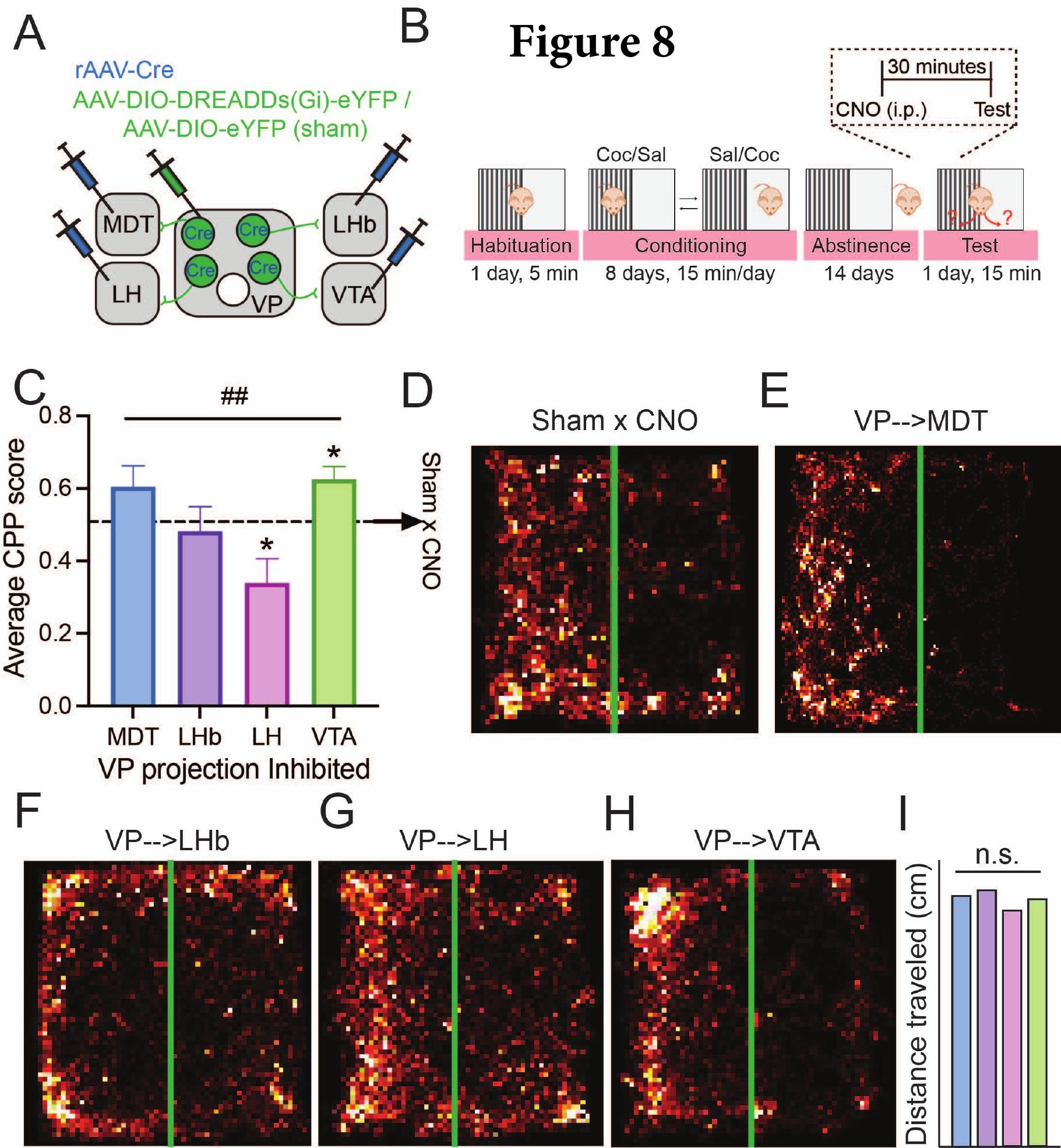

